\title{
Germanica
}

\section{Henriette Roland Holst ou la mort arraisonnée}

Henriette Roland Holst of de aan banden gelegde dood

\section{Gilbert Van de Louw}

\section{OpenEdition}

Journals

Édition électronique

URL : http://journals.openedition.org/germanica/1944

DOI : 10.4000/germanica. 1944

ISSN : 2107-0784

\section{Éditeur}

Université de Lille

\section{Édition imprimée}

Date de publication : 31 décembre 1996

Pagination : 67-82

ISSN : 0984-2632

\section{Référence électronique}

Gilbert Van de Louw, « Henriette Roland Holst ou la mort arraisonnée », Germanica [En ligne], 19 | 1996, mis en ligne le 04 juin 2013, consulté le 06 octobre 2020. URL : http://journals.openedition.org/ germanica/1944; DOI : https://doi.org/10.4000/germanica.1944

Ce document a été généré automatiquement le 6 octobre 2020.

(c) Tous droits réservés 


\title{
Henriette Roland Holst ou la mort arraisonnée
}

Henriette Roland Holst of de aan banden gelegde dood

\author{
Gilbert Van de Louw
}

1 Doit-on s'étonner de voir que Freud, dans sa Correspondance, appelle Multatuli poète, voire "poète hollandais»? L'auteur est surtout connu pour ses contes, sa correspondance et Max Havelaar, premier roman anti-colonialiste de la littérature mondiale dont la traduction allemande a beaucoup contribué à le faire connaître sur le plan international. C'est d'abord une conscience, un homme libre qui utilise le genre littéraire pour dénoncer: dénoncer le système colonial des Indes et le travail des courtiers hollandais, dénoncer le décalage entre la vérité de la métropole hollandaise et les pratiques par delà les frontières. Dénoncer, finalement, le décalage entre un Verbe magnifié par la Nation et une pratique sociale et quotidienne qui n'y avait que de lointains rapports.

Dans cette optique, Multatuli est poète au même titre que Vondel au XVII ${ }^{e}$ siècle, « le prince des Poètes » comme on l'appelait, et Henriette Roland Holst (1869-1952) en ce début de siècle. Ces auteurs pour lesquels la critique utilise volontiers les superlatifs sont en quête d'une fusion entre la vérité à laquelle ils croient et une pratique sociale dont ils estiment qu'elle doit être le reflet de valeurs philosophiques, éthiques et morales. Dans les trois cas aussi, la position du « poète » l'amène à s'aliéner peu ou prou par rapport à ce qui l'entoure, à tout ce qui le fixe sur le plan social : Multatuli s'exilera, Vondel retournera sur le tard au Mont de Piété pour y gagner sa vie comme comptable, Henriette Roland Holst ira de déchirement en déchirement, au fur et à mesure que les idées pour lesquelles elle se bat prennent forme et se déforment par rapport à un idéal, forcément utopiste. Mais dans ces difficiles rapports du créateur au public, ces Hollandais, à tout prendre, rejoignent les autres, Rembrandt, Van Gogh, Van Goens ${ }^{1}$ et tant d'autres Busken Huet. Dans cette optique-là, Freud a raison de parler de "poète hollandais » : la seconde moitié du XIX ${ }^{e}$ siècle voit, en effet, éclore un renouveau culturel qui renoue à bien des égards avec la tradition littéraire du « Siècle d'Or », qui doit son ampleur au mêmes causes et qui fait percevoir la littérature hollandaise à 
l'étranger dans une optique similaire : Domela Nieuwenhuis dont l'action anarchiste fut exemplaire en Hollande ${ }^{2}$ apparaît comme une autorité littéraire à l'étranger. Ses œuvres traduites dans plusieurs langues relèvent pour les éditeurs étrangers de la littérature hollandaise. Or, il s'agit ici d'un pasteur qui - comme bon nombre d'autres se voit confronté dans son quotidien aux décalages entre le Verbe porteur d'espoir et la réalité sociale dans laquelle les idées socialistes se propagent de plus en plus. Ce sont ces réalités du quotidien qui l'amènent à prendre des positions radicales. Son œuvre sera « littéraire " par la réflexion qu'elles présentent, une réflexion dans laquelle la rhétorique spéculative le dispute à la philosophie. Elle sera littéraire aussi parce qu'elle incarne - comme ce fut le cas des œuvres de Huyghens ou de Hooft, deux siècles plus tôt - les rêves d'une Nouvelle Israël. Certains auteurs des années 480 , connus comme les Tachtigers, "ceux des années Quatre-Vingts ", croient que les idées socialistes vont régénérer l'espace politique et littéraire des Pays-Bas: pour eux, le pays va être un vaste champ d'application de théories nouvelles vécues comme autant de religions et les créateurs - que ce soient des littéraires ou des artistes - se doivent de s'en inspirer pour donner forme et consistance à la vie de l'esprit dont ils sont porteurs et garants.

Henriette Roland Holst est exemplaire des paradoxes d'une telle situation et des engagements « à la hollandaise » des auteurs. Elle naît en 1869 dans la province de la Hollande septentrionale, dans un milieu aisé et libéral. Son père est notaire. La famille appartient à l'Église réformée, celle qui est ouverte aux temps modernes, aux idées nouvelles sur ce que doivent représenter l'État et la Nation. Rappelons qu'à cette époque-là on connaît aussi un "retour aux sources » collectif qu'incarne Abraham Cuyper, fondateur en ces années-là de l'ARP, Anti-Révolutionnaire Partij, qui vise à restaurer une société conforme aux idées d'« avant-révolution », c'est-à-dire d'avant la Révolution française qui, libérale, préconisait une égalité de citoyen, peu compatible avec l'idée de prédestination. Conformément aux engagements religieux de son enfance et en dépit de ses choix parfois radicaux, l'auteur voit dans la figure du Christ le symbole spirituel et moral de l'Europe de l'Ouest et ne rompra jamais tout à fait ses liens avec l'église reformée : même après avoir renoncé à en être membre, pour des raisons secondaires, elle continuera à considérer que la déclaration de principe de cette communauté est « magnifique $»^{3}$.

Dans ce climat d'une liberté qui se redéfinit et d'une société qui se cherche, Henriette Roland Holst fera plusieurs rencontres décisives : celle du peintre Jan Toorop qui, issu des Indes, la confronte avec un autre monde, tant par les sentiments que par sa démarche symboliste. C'est l'univers d'une quête de vérité par delà les conventions sociales et le climat quelque peu engoncé de cette Hollande des traditions protestantes. Elle note elle-même, dans ses souvenirs ${ }^{4}$, qu'à la vue des tableaux de l'artiste, «le monde du beau rêve s'ouvrit enfin » devant elle, alors qu'elle se voit confrontée à des œuvres "devant lesquelles j'étais étrangère " ${ }^{5}$. C'est Toorop qui la mettra en contact avec Albert Verwey, le mentor qui l'encouragera dans la voie de l'écriture littéraire. C'est Herman Gorter enfin, qui l'amène (fin 1893) à lire aussi bien Dante que Spinoza à la suite de longues discussions philosophiques et qui l'engage sur la voie d'une écriture exposée aux tourments d'une vie de convictions.

On peut s'étonner de voir un tel engagement, surtout par rapport au milieu dont elle est issu. La mort accidentelle d'un père en 1892 n'y est certes pas étranger, pas davantage, d'ailleurs, que sa rencontre avec son futur époux. Son mariage avec le peintre R.N. Roland Holst - admirateur de William Morris, l'artiste et socialiste anglais 
- l'a certainement amenée à considérer l'expression littéraire dans un ensemble plus vaste dont témoignent les titres de ses œuvres: de Sonnetten en Verzen in terzinen geschreven (Sonnets et Vers écrits en terzines), son premier recueil datant de 1893 qui fut aussi l'année de sa rencontre avec son futur mari, à Nieuwe Geboort (La Nouvelle naissance) de 1903, il y a l'émancipation d'une femme qui reconnaît elle-même qu'elle est « autodidacte, comme la plupart des prolétaires ${ }^{6}$ et qui s'est ouverte au monde. Un passage à vide dans son inspiration poétique, une prise de conscience politique à travers les articles de Tak et l'engagement de son mari vis-à-vis de l'œuvre de Morris semblent les principales raisons d'une révolution de l'esprit :

Mon mari était très touché par la mort de Morris ; il sortit ses œuvres de la grande bibliothèque surmontée d'un hibou et nous nous mîmes à relire ses essais sur l'art et la société que nous n'avions pas repris depuis des années ;

Apparemment j'avais assez mûri dans ces années-là pour les comprendre. C'est comme si les yeux se désillaient, comme si je voyais d'une lumière nouvelle et éclatante la société et la vie qui m'entouraient. Le capitalisme non seulement avait condamné les masses à une vie sans joies, vie de peine, d'ignorance et de déchéance, il avait aussi enlaidi le monde, assassiné la beauté de la nature, comme celle des vieilles cités et tué l'idée de sentiment collectif, sans lequel l'art ne saurait exister?

6 Tout est ainsi mis en place pour que socialisme rime avec « renouveau culturel». L'auteur a fait le saut dans le vide, laissé derrière elle la tradition parce qu'elle a découvert la vie. Et, comme Theo van Doesburg dans la Préface à l'art moderne, elle plaide pour le mouvement, signe vivant de la théorie à laquelle elle croit: "Théorie et mouvement, principes et vie ne sont pas des contrastes, ne sont pas contradictoires et celui qui les oppose retombe dans la manière de penser traditionnelle, dépassée, figée. Le principe n'est donc pas comme un gardien ennuyeux entre la social-démocratie et la vie ; c'est un guide et un lamaneur qui indique dans la vie la voie pour atteindre le but final de la manière la plus rapide en suivant les voies les plus sûres et les plus courtes » 8 .

7 Henriette Roland Holst rompt donc avec la classe à laquelle elle appartient de par son milieu familial. Elle fera de la défense des opprimés l'affaire de sa vie, comme si la conviction qu'elle mettait à cette cause-là tenait lieu de droit sacré.

8 Sa vie sera dorénavant sous le signe de cet engagement-là, à travers les vissicitudes du socialisme européen. Elle s'engage dans le SDAP (Sociaal-Democratische Arbeiders Partij) en 1897 et mettra à la disposition de cette cause, son talent, sa persuasion, son intelligence et son cœur. Radicale, elle le sera, au nom de ce choix, radicale dans ses options marxistes, radicale dans son engagement de tous les jours, radicale encore au moment des choix définitifs : la scission du SDAP lors du congrès de Deventer (1909) signifie pour elle la fin tragique de l'unité du mouvement ouvrier, autant que de son amitié avec Herman Gorter qui devient un des chefs de la SDP (Sociaal Democratische Partij) auquel elle n'adhère pas. En 1911, elle quittera le SDAP.

9 Ce n'est pas pour autant la fin de ses activités politiques : la guerre la voit sur le front de l'aide humanitaire aux réfugiés dans cette Hollande neutre. Comme révolutionnaire et anti-militariste, elle participe à la conférence de Zimmerwald (sept. 1915) «point lumineux dans la nuit noire pour des millions de personnes dans les tranchées »9 . C'est pour elle l'occasion d'approfondir ses relations avec Trotsky qui est pour elle le héros de Brest-Litowsk, « le constructeur, le géniteur, celui qui donne forme et qui purifie ${ }^{10}$ et de participer activement à la rédaction du manifeste final, dans lequel la solidarité 
humaine est affirmée avec force. De retour en Hollande, elle participera activement aux réunions publiques en faveur de la paix, conformément aux décisions prises en Suisse :

Mais le combat pour Zimmerwald (i.e. les décisions qui y avaient été prises) était très différent; c'était la solidarité avec des millions de Français, d'Anglais, d'Allemands, d'Autrichiens, de Russes qui souffraient dans les tranchées de faim et de soif, exténués et angoissés, qui étaient déchiquetés par les grenades et ensevelis sous les décombres. C'était l'amour pour tous ceux qui avaient gardé leur sentiment d'homme, donnaient de leur propre gourde à boire à un ennemi mourant ou promettaient de renvoyer à l'adresse indiquée le petit portrait qu'il portait sur son cœur $^{11}$.

La révolution russe fera d'elle une communiste :

Pour la première fois depuis la Commune, la classe ouvrière pouvait organiser la société d'après ses propres conceptions. Mais cette société était complètement défaite, ruinée et chaotique. Il fallait une force titanesque pour la réorganiser, ne fût-ce qu'un petit peu ${ }^{12}$.

11 Communiste, elle le restera jusqu'en 1927. L'enthousiasme du choix et d'un parti en harmonie avec ses aspirations profondes s'estompe rapidement devant le problème de la violence, qu'un voyage à Moscou (1921) ne fera que rendre plus aigu. Par ailleurs, même si elle comprend la nécessité d'une discipline de parti, elle s'y soumet de plus en plus difficilement, devant des évolutions politiques et économiques éloignées de l'idéal dont elle se sait porteur.

Verworvenheden (Acquisitions) de 1927 et Vernieuwingen (Renouveau) de 1929 montrent un retour vers les valeurs religieuses. Faut-il y voir «la confession de ses erreurs et errements » comme le prétend K.-F. Proost ${ }^{13}$ plutôt qu'une réflexion sur la fonction du poète et le constat que l'action est nécessairement relative? N'est-ce pas plutôt une réorientation dans sa vie qui l'amène à privilégier les choix de l'individu plutôt que de la collectivité ? Des choix de poète, parce que, justement, celui-ci est révolutionnaire à sa manière?

13 Poète, elle le sera donc à travers des œuvres de nature diverse. Les discours d'abord, œuvres d'engagement qu'elle adresse à toutes sortes de groupes sociaux, comme pour les instruire. Elle répond aux nombreuses sollicitations et publie de nombreuses contributions dans des revues dont le public était plus ou moins large. Comme d'autres avant elle - les sœurs Bronte par exemple - son engagement est militant. Notons, dans ce cadre, les nombreux titres destinés aux femmes et à leur monde, celui de l'éducation, de leurs droits, de leur spécificité : Vrouwen, luistert eens, De Vrouw, de Arbeidswetgeving en de Sociaaldemocratie, De Moeder, Der Vrouwen Weg, De Vrouw en het Communisme.

Les études, ensuite, parmi lesquelles Kapitaal en Arbeid in Nederland de 1902, constamment élargi jusqu'à la quatrième édition de 1932 ou Communisme en Moraal de 1925. Ces œuvres frappent par le souci de placer les évolutions politiques et sociales dans une perspective tantôt plus morale tantôt plus éthique. Ces engagements ne diminuent en rien les exigences littéraires du poète dont font preuve les titres qui, traditionnellement, se rangent parmi les œuvres littéraires.

15 Socialisme ou pacifisme deviennent source d'inspiration et d'évolution dans $D e$ Opstandelingen, Lyrisch Tereurspel in drie Bedrijven (Les Insurrectionnels, cuvre lyrique de la Terreur, en trois actes), drame de la révolution avortée de Russie en 1905 ou encore dans Thomas More (1912) où elle présente le chancelier comme le héros du refus, précurseur de l'idée communiste. A ce titre il importe de constater qu'Henriette Roland Holst est poète dans la mesure où elle aborde aussi bien la tragédie, comme Vondel le fit, que la 
poésie plus personnelle dont témoignent De Vrouw in het Woud (1912) ou Verzonken Grenzen (1918), De Stem die roept (1936), De loop is bijna volbracht (z.j., sans date). Ces différentes œuvres reflètent des évolutions qui sont autant d'engagements personnels dans lesquels le poète chemine sur la voie de l'exemplaire solitude. Et ce faisant, il devient une voix.

Que signifie, dès lors, le mot "engagé " pour le poète hollandais de cette première moitié du $\mathrm{XX}^{\mathrm{e}}$ siècle? Peut-on, avec Garmt Stuiveling, conclure à une "poésie prophétique » :

En dépit des changements dramatiques survenus en 1897, 1911 et 1927, qui lui ont été fortement reprochés de part et d'autre, sa vie montre une grande unité, dès lors qu'on la voit inconditionnellement au service d'une poésie prophétique ${ }^{14}$.

17 Est-ce dès lors la poésie qui prime ou l'élément prophétique, celui-là même que les Nabis ont choisi comme signe distinctif de leur groupe ? J.-P. Van Praag, dans Henriette Roland Holst wezen en werk ${ }^{15}$, met en évidence pour sa part une vie "dirigée par une puissance dont elle avait su, dès son enfance, l'arrivée - le motif d'espérance ». Le poète lui-même reconnaît la difficulté de ces « changements » quand elle relate son retour de Gland après un détour qui lui a permis de rencontrer Romain Rolland :

Je rentrai fatiguée, mais intensément heureuse. «Je sais à présent où est ma place et comment on peut vivre heureux » dis-je à mon mari. « Je suis heureux de savoir que tout s'est bien passé et que les gens étaient aimables. Mais tu as changé de cap si souvent » ajouta-t-il avec un soupir. «J'espère que tu as trouvé à présent quelque chose de permanent $»^{16}$.

18 Les mots sont retors, mais sont tous - "prophétique ", "motif d'espérance » - des projections dans l'avenir. S'agit-il d'utopies? Que chercher derrière cette "espérance ", sinon une abstraction? Et qu'en est-il de cette espérance en pays protestant? Quelle peut être la signification de cette notion dans un pays secoué par des querelles dans lesquelles, constamment, la référence au « decretum horribile » fait la différence ? Et quel peut être le sens littéraire du mot «verwachting», quand, chargé de vie et de vitalité, il s'applique aux femmes enceintes, porteuses de vie et donc «in verwachting» (enceintes) ? Quand, de surcroît, il s'applique à une femme-poète dont le mariage reste sans enfants?

Ces différentes raisons militent en faveur d'un nouvel examen du «cas " Henriette Roland Holst dont on a trop souvent mis en évidence les inconséquences plutôt que la cohésion interne et dont, tout au plus, on pourrait mettre en évidence des cheminements dans lesquels l'action prend plus ou moins le pas sur la réflexion poétique. Cette action génère une activité littéraire importante. Sans cette action, sans ses déplacements en Russie (1921) et ailleurs, qu'en serait-il advenu de ses idées révolutionnaires qui ont tant nourri sa réflexion et sa poésie? Ses déplacements sont autant de voyages dans ce «mouvement» pour lequel elle a opté dès ses débuts, comme pour la raison d'être de sa condition de poète. Le choix initial était révolutionnaire, générateur d'un engagement de tout instant au service d'une cause. C'est cette cause qui fait accoucher le poète d'une réflexion philosophique et morale, c'est par elle que se forgent les Idées. C'est elle qui engendre les Images du vrai poète. C'est la pérennité de la cause qui jugule l'idée de temps et de mort dont cette Hollande est si familière.

20 On peut, en effet, se demander quelle est l'unité de cet œuvre qui est aussi une vie. Henriette Roland Holst est d'abord libre. Elle a acquis cette liberté par sa recherche d'une vérité, les choix et engagements « sur le terrain » qui en ont résultés. Elle justifie 
par cette liberté sa conduite, dans la période la plus difficile de sa vie, quand elle ne peut pas combattre la violence des appareils d'Etat :

Mon mari et moi-même sommes passés par des moments difficiles à mon retour (de Russie). Il s'est évidemment rendu compte que mon séjour en Russie soviétique avait été un désenchantement pour moi. Il avait espéré que je me détournerais du communisme et ne comprenait pas que ce n'était pas mon idée. Je ressentais toujours la vérité des paroles de Rutgers: «Le comintern est la communauté mondiale et pacifique des opprimés ». Et tant que j'avais ce sentiment, je n'avais pas la liberté de me retirer. ${ }^{17}$

21 La liberté est donc un choix, dicté par une raison, elle-même tributaire d'options qui relèvent de l'ordre de la foi. C'est cette liaison entre liberté et foi qui fonde la démarche intellectuelle. Dans les années 1920 elle écrira :

La conception qu'on a à Moscou repose toujours sur la vieille répartition des gens en deux catégories : une élite restreinte et une multitude de gens qui suivent. C'est cette répartition qui domine le passé ; on est fondé, pour toutes sortes de raisons, à douter de sa disparition dans l'avenir. C'est, toutefois, la vocation du socialisme dans ce qu'il a de plus élevé de la réduire au maximum en amenant les masses à chercher, juger et se suffire par elles-mêmes ${ }^{18}$.

La raison elle-même devient de l'ordre religieux, puisqu'elle amène l'auteur à une Idée dont l'essentiel est d'ordre religieux et mystique, celui de l'ordre du partage. Religieuse, elle le sera par la quête d'une unité de l'homme et de l'univers, unité qui n'est pas fondée sur l'idée d'un Dieu personnel. À partir de cette conception, elle se veut expressément « religieuse » surtout dans ses années d'engagement communiste. Mais à l'intérieur de cette conception "dynamique » d'une foi dans la collectivité, elle évolue d'un "salut de l'ensemble ou de l'unité sociale la plus étendue, à savoir la classe " (1915) vers la reconnaissance des spécificités : «les actions de la classe prolétaire se jugent bien par leur valeur particulière et spécifique, par rapport au processus général de l'humanité » $(1925)^{19}$

En cet hiver 1921-1922 elle se verra confrontée aux réalités concrètes de ses choix. Cet hiver terrible pour elle, parce que les morts russes s'amoncellent. Mais devant l'immensité de la catastrophe, elle aligne les chiffres: «Il fallait que dix millions d'hommes, de femmes, d'enfants meurent, mais vingt millions furent sauvés $»^{20}$. L'action est encore, pour elle, l'expression la plus pure de l'amour humain ${ }^{21}$.

Le poète trouve sa place dans cette évolution parce qu'il est porteur d'un idéal dynamique qu'il contribue à faire connaître par l'inspiration de son art. Dans ce sens, sa fonction ne diffère pas de celle de ses aînés hollandais. C'est le concept et le rôle de l'art qui ont évolué dans la perception des hommes. Pour Henriette Roland Holst, cet art est celui du partage, celui de la communauté - gemeenschapskunst. Elle a, dans sa longue carrière, évolué dans les mots, mais le principe est identique. Qu'elle l'appelle « art du partage" ou "religion", voire "amour", elle se réfère toujours à cette unité universelle, à laquelle on accède par le partage d'un art qui se diffuse par l'adhésion collective et communautaire. La fonction de cet art se définit ainsi :

Purification des passions aveugles, épure des pulsions aveugles, de la douleur aveugle, de la superbe aveugle, voilà depuis toujours la vocation la plus élevée de la tragédie. Les grands tragiques ont toujours essayé de susciter auprès de leurs spectateurs l'idée que l'homme le plus fort est grand quand il se sent partie de la Vie infinie; l'idée que la suffisance et la superbe sont opposées aux forces les plus profondes de cette Vie, que l'esprit de conciliation, de générosité et de modération sont les clefs d'or qui ouvrent les portes qui mènent à la solution des douloureuses 
contradictions de l'existence humaine. La force de la tragédie vient de ce que l'on vit ces sentiments comme vérité suprême dans toute sa pureté. ${ }^{22}$ (c'est péché) pour dire qu'une chose est simplement regrettable. Pour ce qui est de la mort, rappelons que ce monde hollandais se façonne par la lecture quotidienne de la Bible, une Bible qui en charrie un usage bien particulier, dans la mesure où elle parle d'« enfants de la mort " pour désigner ceux qui sont dans le péché. La structure de la langue néerlandaise est affectée par cette mort perçue comme un au-delà moral des frontières de la vie, c'est-à-dire un monde, par delà le perceptible où elle règne en souveraine. Peut-on dire, par comparaison au français, qu'elle y est en même temps plus banalisée et davantage acceptée dans sa réalité immuable, alors que les expressions françaises témoignent d'une préférence pour les aspects actifs - qui sont autant de négations implicites de la mort et privilégient plutôt l'action de mourir: «mourir de rire», de ce point de vue n'est pas l'équivalent de «zieh doodlachen» et l'étranger reste abasourdi devant des mots comme «doodleuk» dans lequel «leuk»a le sens habituel de «sympa ». «Doodeenvoudig» est tout bonnement «très simple » et montre que le préfixe «dood» finit par renvoyer à une intensité prononcée, sorte de superlatif descriptif. Dans des expressions idiomatiques la mort garde cette ambiguïté : «als de dood zijn voor iets» est davantage concret dans son équivalent français "craindre quelque chose comme la peste». Le substantif «dooddoener» «formule banale » montre bien qu'elle constitue une frontière par rapport à laquelle l'homme se mesure en tant que sujet agissant. Les dictons populaires en portent la trace dans des expressions telles «de een zijn dood is de ander zijn brood» « ce qui fait le bonheur des uns fait le malheur des autres ». L'adjectif «doods» renvoie à « morne » ou à « désert ", comme pour mieux souligner que c'est la présence humaine en tant qu'elle donne vie qui détermine le sens, présence humaine d'autant plus difficile à détecter : là que les comparaisons avec le français sont parfois paradoxales : la «nature morte» l'est parce qu'il n'y a pas de présence humaine, alors que les Hollandais considèrent qu'il s'agit d'un «stilleven», parce que le tableau est une construction " parlante », même si elle est dépourvue de présence humaine... Ces différences dans l'appréciation de la conception de ce qui est humain amènent, d'ailleurs, à des modifications de certains tableaux: Ruysdael, par exemple, aurait pu voir ainsi son Champ de Blé meublé de bergers s'il avait vécu au XVIII siècle, probablement parce que les amateurs considéraient à l'époque cette présence nécessaire dans ce qu'ils considéraient comme une "scène bucolique " !

Pourtant, la langue montre clairement que la mort ne se cantonne pas au physiquement vivant et humain : «een dode blik» est « un regard sans vie », celui d'un fou, par exemple, ce qui montre bien que « vie » et « mort » se définissent à travers des concepts culturels. Pourquoi, sinon, aurait-on recours à des formules telles «mort clinique ", si ce n'est pour signifier que l'on parle d'une autre mort?

Henriette Roland Holst agit sur ce concept culturel de mort, comme pour déjouer la Mort. C'est dans ce sens que l'on peut la considérer comme révolutionnaire dans un contexte hollandais, chargé d'angoisse et de culpabilité. Elle est entière dans son action militante, parce que, justement, c'est aussi une échappatoire que la raison construit pour déjouer cet inéluctable rendez-vous avec soi-même. L'action n'est pas un but en 
soi. C'est une traduction qui reste inscrite dans la poétique. Comme les Tachtigers, elle utilise la puissance, ainsi, les métaphores marines, mais ses Idées s'inspirent davantage d'une rhétorique spéculative modernisée par les références à la réalité sociale que de la philosophie. C'est ce qui donne à ses métaphores une densité particulière, reconnue d'ailleurs par Marsman lui-même ${ }^{23}$. Dans ce travail sur les «beeiden», ces «vues » d'un esprit plutôt que de l'esprit, elle fait passer la passion d'une femme préoccupée par ce qu'elle considère comme essentiel : la place précaire de l'homme entre liberté et liens de tous ordres, qui fondent sa nature éthique.

Son engagement social ne l'aveugle pas, cependant, comme le montre Les Insurrectionnels de 1910. Bien avant qu'elle ne se décide à tourner le dos aux communistes, elle en arrive à des constats bien mortifères. L'œuvre donne la clef pour comprendre pourquoi elle s'est engagée et ce qu'elle pense de l'action révolutionnaire : cette tragédie lyrique est tragique, parce que la révolution russe de $1905 \mathrm{y}$ apparaît inévitable autant que son issue et son échec. C'est le Verbe et le style, «sublimation de la réalité $»^{24}$, qui justifient l'engagement du poète, un style si particulièrement baroque dont Garm Stuiveling a étudié le rythme et la musicalitée 25 .

Le style, cependant, ne peut tenir lieu de philosophie ou de rhétorique, même spéculative. Et si Henriette Roland Holst semble se détourner des habitudes et courants littéraires traditionnels, elle doit une large part de sa force créatrice à ses propres combats - qu'ils soient extérieurs ou intérieurs, engagés pour tous parce qu'elle veut que tous connaissent la joie du partage ou pour elle-même, dans la mesure où elle veut échapper à cette pulsion de mort, dont Freud commence à décrire les effets sur la psyché humaine. C'est pourquoi elle évoque souvent la mort recourant aux métaphores marines, comme dans «diepe baai en altijd open haven» (Baie profonde, havre toujours accessible), où la mort apparaît de façon très plastique comme cet endroit en même temps profond et "sûr ", lieu mystérieux puisqu'insondable en même temps que familier et sécurisant puisque «baie». C'est par le travail qu'elle fait sur les métaphores qu'elle crée une poésie synonyme d'une paix intérieure lentement conquise. La mort s'oppose finalement à la violence dont l'homme subit trop souvent la dure loi, dans sa vie personnelle ou dans les comportements collectifs : «Ik wil weer wonen in de groote vree» (Je veux vivre, à nouveau, dans la grande paix) dit-elle dans De Vrouw in het Woud. Dans Nieuwe Geboort, elle place un poème avec le titre évocateur : «Leeg te zijn van Wenschen als in den Dood» (être vide de désirs comme dans la Mort). Tussen îwee werelden également présente la mort comme libération des pulsions et des désirs. Le poète constate que cette mort n'est pas plus cruelle que la vie, pas davantage mystérieuse. C'est qu'elle intègre, dans sa réflexion, cette mort, à partir du devenir d'un temps relativisé qui réduit la mort à n'être qu'un enfant : on le porte, tout autant que les idées, les actions et les désirs :

Je veux vous appréhender davantage, ô Mort

telle que vous grandirez en moi comme un enfant.

N'êtes-vous pas notre enfant, quand le soleil du soir

se charge d'ombres et que naît le crépuscule?

Quand alors vous vous présenterez, hôte longtemps attendu

de nos idées et nos rêves,

ne serai angoissé ni surpris

mais vous saluant d'un "bien venu »

vous suivrai vers les portes, que votre main

libère - les portes sombres derrière lesquelles 
s'étend le pays infini

chargé de brumes, dont vous êtes le gardien ${ }^{26}$. hollandais par cette quête de vérité et d'une harmonie cosmique fondée sur des critères éthiques et des convictions, de son temps, par son engagement socialiste à un moment où ce choix n'était pas encore celui d'une société, mais celui d'une vérité humaine et collective. Elle s'est engagée dans ce combat avec tout le poids de sa fonction de poète, à l'instar d'autres auteurs ou artistes qui, tantôt « suicidé de la société » comme ce fut le cas pour Van Gogh aux dires d'Artaud, tantôt chantre du monde de la mer, comme ce fut le cas pour le neveu d'Henriette Roland Holst, ont cherché à donner sens à un Verbe qui les met au diapason d'un au-delà de l'angoisse de mort.

\section{NOTES}

1. - Voir G. Van de Louw, « R.M. van Goens (1748-1810), correspondant patriote ou cosmopolite », in L'art épistolaire dans l'Europe cosmopolite, Cahiers d'histoire littéraire comparée, $\mathrm{n}^{\circ} 11$, Paris, Didier Erudition, pp. 111-122.

2. - Henriette Roland Holst note dans ses souvenirs «Les sermons de Domela Nieuwenhuis avaient sorti le prolétariat de sa torpeur, du moins à Amsterdam et en Frise. Et lorsque le chef, aimé, voire idolâtré, fut condamné à un an de prison parce qu'il avait parlé du Roi Guillaume III en termes peu convenables, l'amertume fut générale. », Het vuur brandde voort, Amsterdam, 1949, p. 37. Henriette Roland Holst lui consacrera quelques notices lors de sa mort, cf. De Nieuwe Tijd, 1919, et lors du vingtième anniversaire de cette mort, cf. Tijd en Taak, 1939, année qui n'est guère innocente.

3. - Cf. Dr J.P. van Praag, Henriette Roland Holst wezen en werk, Amsterdam, 1946, Uitg. Contact, 220 pp., p. 37.

4. - Henriette Roland Holst-van der Schalk Het Vuur brandde voort Levensherinneringen (Le feu continuait de brûler, souvenirs d'une vie), Amsterdam, v.h. Van Ditmar, 287 pp., p. 57-58.

5. - Ibid, «werken waar ik vreemd tegenover stond».

6. - «Evengoed als de meeste Proletariers ben ik sedert 1892 autodidact», Het vuur brandde voort, p. 37. Rappelons, pourtant, qu'elle avait suivi des études dans un pensionnat «bien sous tous rapports » et avait passé un hiver à Liège, pour parfaire son français.

7. - Idem, p. 95 «Mijn man was zeer getroffen door de dood van Morris».

8. - Cf. son article «Beginsel en Praktijk» in De Nieuwe Tijd, VII, 1902, pp. 195 : «Theorie en beweging, leer en leven, zijn geen contrasten, niet in strijd met elkaar, en wie ze tegenover elkaar stelt vervalt in de oude, overwonnen, starre denkwijze. Het beginsel Staat dus niet als een lastige wachter tusschen de sociaal- democratie en het leven, maar het is de gids en de loods die in het leven den weg wijst om zoo snel mogelijk langs de veiligste, kortste banen het einddoel te naderen».

9. - Het vuur brandde voort, p. 158.

10. - Heldensage, 1927, «de bouwer, de bevruchter, de vormgever, de grote zuiveraar», cité par Van Praag, op. cit., p. 30.

11. - Idem, p. 166.

Germanica, 19 | 1996 
12. - Idem, p. 170.

13. - Dr K.-F. Proost, Henriëtte Roland Holst in haar strijd om gemeenschap, Arnhem, van Loghum Slaterus, 1937, 288 pp., p. 280, «de belijdenis van haar vergissingen en dwalingen».

14. - Notice biographique. Moderne Encyclopédie van de Wereldliteratuur, $2^{\mathrm{e}}$ éd.. De Haan, Weesp, De Standaard, Anvers, 1983, vol. 8, p. 152a, «Ondanks de dramatische wijzigingen in 1897, 1911 en 1927 , die haar van versch. zijde tel zijn verweten, toont haar leven een grote eenheid zodra men het ziet als onvoor-waardelijke dienst aan een profetisch dichterschap.»

15. - J.-P. Van Praag, op. cit., p. 78.

16. - Het vuur brandde voort, p. 220.

17. - Idem, p. 214.

18. - De Nieuwe Tijd, 1920 et De Taak der Commimistische Partij in de Proletarische Revolutie, cités par van Praag, op. cit., p. 31.

19. - Cf. van Praag, op. cit., p. 33. La première citation est de De Nieuwe Tijd, «Proletarisch belang en proletarisch ideaal», «het heil van de grootst moge- lijke sociale eenheid of algemeenheid, d.i. de klasse», la seconde est tirée de Communisme en Moraal, «de daden van de proletarische klasse pas hun eigenaardige en bijzondere waarde ontlenen aan hun betrekking tot het algemeene levensproces der menschheid».

20. - Idem, p. 215 «Tien inillioen mannen, vrouwen en kinderen moesten sterven, maar twintig millioen werden gered».

21. - Ibid. : « Nansen kwam op zijn doorreis ook te Amsterdam. Ik hoorde hem spreken: rüstig, beheerscht, bezielend. En toen ik hem de hand drukte, wist ik, dat één ding elke politieke overtuiging te boven gaat en dat is zuivere menschenliefde».

22. - Over Dramatische Kunst, p. 269 : «Reiniging van blinde hartstocht, loutering van blinde driften, blinde smart, blinden hoogmoed, dat was altijd de hoogste roeping der tragedie. Altijd poogden de groote treurspeldichters in de toeschouwers het besef te wekken, dat de Sterke mensch het grootst is, wanneer hijzieh een deel voelt van het oneindig Leven; het besef dat overmoed en hoogmoed tegen de diepste krachten van dat Leven ingaan, dat verzoenlijkheid, mildheid en matiging de gouden sleutels zijn, die de poort openen tot de oplossing der smartelijke tegenstrijdigheden in het menschelijk bestaan. In het als hoogste waarheid beleven van deze gevoelens lag altijd der tragedie zuivere kracht».

23. - Cf. Marsman, Critisch Proza in Verzamelde Werken.

24. - Cité par Van Praag, op. cit., p. 50. Le poète utilise l'expression dans une recension à propos d'un drame, justement.

25. - G. Stuiveling, Versbouw en Ritme in de Tijd van '80, 1934, chap. XIII.

26. - « Ik wil u al dieper indenken, dood, / zóó dat ge in mij groeit gelijk én kind./ Zijt ge ons kind niet, als het avondrood/ vervaluwt en de schemering begint? /// Als ge dan komt, de langverwachte gast/ van onze waakgedachte' en onze droomen, / zal ik niet angstig zijn, zelfs niet verrast, maar, u begroetend met een "welgekomen», /// u volgen naar de poort, die uwe hand ontgrendelt, - de donkere poort waarachter/ gespreid ligt het oneindig land, / nevelbedekt, waarvan ge zijt de wachter.», in Tussen Tijd en Eeuwigheid. 


\section{RÉSUMÉS}

Henriette Roland Holst s'affranchit très tôt d'un milieu protestant, c'est-à-dire d'un monde qui identifie le péché au domaine de la mort, dans la tradition biblique. Elle découvre la beauté de l'art et l'étrange monde de la peinture et de la littérature, une "autre » réalité si vraie et si éloignée du quotidien. Elle s'oriente vers la littérature « engagée » où le poète parle au service de tous, mais incarne, aussi, un espoir «raisonnable»: l'engagement est rationnel dans sa démarche, mais éthique par les choix.

Les critiques voient dans la vie et l'œuvre du poète deux phases distinctes, celle de l'engagement politique au service du socialisme, du pacifisme et de l'émancipation de l'être humain et celle du retour sur soi, après la déception, quand elle aborde davantage la création d'un point de vue mystique. Une telle dichotomie ne tient pas assez compte des réalités de la création poétique et des thèmes abordés: Henriette Roland Holst s'approprie le socialisme comme un monde de raison et d'espoir, un univers où l'homme trouve la justification de son combat, où la mort ne correspond plus à une idée de péché, mais devient l'aboutissement de choix qui, seuls, peuvent délivrer l'homme des pensées mortifères parce qu'ils arraisonnent la mort à des convictions qui ont guidé la vie.

Henriette Roland Holst bevrijdt zieh al vroeg van een protestante omgeving, van een wereld dus waar zonde «des doods» is. Ze ontdekt de schoonheid van de kunst en de vreemde wereld van schilderkunst en literatuur, een «andere» realiteit, zo ver van het dagelijkse. Ze kiest voor een geëngageerde literatuur waar de dichter voor ieder spreekt maar ook een «redelijke» hoop voorstelt: zij is rationeel in haar ontwikkeling, ethisch in haar keuze.

De kritiek heeft vaak in haar leven twee verschillende phasen gezien, de eerste in dienst van het socialisme, het pacifisme en de emancipatie van de mens, de tweede meer een mystische inkeer na ontgoocheling. Een dergelijke tweespalt houdt geen rekening met de thema's die zij aanroert en met de waarheid van de dichterlijke creativiteit: Henriette Roland Holst maakt zieh het socialisme eigen als een wereld van rede en hoop, waar de mens vecht voor zichzelf, waar de dood niet meer met «zonde» overeenkomt, maar het resultaat is van keuzes die alleen in Staat zijn de mens te bevrijden van mortifère gedachten, omdat zij die doodsgedachte inbedden in overtuigingen die leidraad worden voor het leven. De dood is dan alleen nog maar het einde van een leven, door de rede beheerst en uitgedragen.

\section{AUTEUR}

\section{GILBERT VAN DE LOUW}

Université Charles-de-Gaulle - Lille III 